\title{
Wadge Degrees of Infinitary Rational Relations ${ }^{\star}$
}

\author{
Olivier Finkel
}

\begin{abstract}
We show that, from the topological point of view, 2-tape Büchi automata have the same accepting power as Turing machines equipped with a Büchi acceptance condition. The Borel and the Wadge hierarchies of the class $\mathbf{R A T}_{\omega}$ of infinitary rational relations accepted by 2-tape Büchi automata are equal to the Borel and the Wadge hierarchies of $\omega$-languages accepted by realtime Büchi 1-counter automata or by Büchi Turing machines. In particular, for every non null recursive ordinal $\alpha$, there exist some $\boldsymbol{\Sigma}_{\alpha}^{0}$-complete and some $\boldsymbol{\Pi}_{\alpha}^{0}$-complete infinitary rational relations. And the supremum of the set of Borel ranks of infinitary rational relations is an ordinal $\gamma_{2}^{1}$ which is strictly greater than the first non recursive ordinal $\omega_{1}^{\mathrm{CK}}$. This very surprising result gives answers to questions of Simonnet Sim92 and of Lescow and Thomas Tho88, LT94.
\end{abstract}

Keywords. 2-tape Büchi automata; infinitary rational relations; Cantor topology; topological complexity; Wadge hierarchy; Wadge degrees; Wadge games; Borel hierarchy; complete sets.

\section{Dedicated to Bill Wadge on the occasion of his 60 th birthday}

\section{Introduction}

In the sixties, automata accepting infinite words were firstly considered by Büchi in order to study decidability of the monadic second order theory S1S of one successor over the integers Büc62. Then the so called $\omega$-regular languages have been intensively studied and have found many applications for specification and verification of non terminating systems, see Tho90, Sta97, PP04 for many results and references. On the other hand, rational relations on finite words were also studied in the sixties, and played a fundamental role in the study of families of context free languages Ber79. Investigations on their extension to rational relations on infinite words were carried out or mentioned in the books [BT70, LS77]. Gire and Nivat studied infinitary rational relations in Gir81, GN84. These relations are

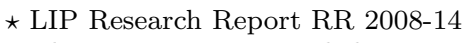

$\star$ This paper is an extended version of a conference paper which appeared in the Proceedings of the 23rd International Symposium on Theoretical Aspects of Computer Science, STACS 2006, Fin06b. 
sets of pairs of infinite words which are accepted by 2-tape finite Büchi automata with asynchronous reading heads. The class of infinitary rational relations, which extends both the class of finitary rational relations and the class of $\omega$-regular languages, and the rational functions they may define, have been much studied, see for example [CG99, BCPS03, Sim92, Sta97, Pri00].

Notice that a rational relation $R \subseteq \Sigma_{1}^{\omega} \times \Sigma_{2}^{\omega}$ may be seen as an $\omega$-language over the alphabet $\Sigma_{1} \times \Sigma_{2}$.

A way to study the complexity of languages of infinite words accepted by finite machines is to study their topological complexity and firstly to locate them with regard to the Borel and the projective hierarchies. This work is analysed for example in [Sta86, Tho90, EH93, LT94, Sta97]. It is well known that every $\omega$-language accepted by a Turing machine with a Büchi or Muller acceptance condition is an analytic set and that $\omega$-regular languages are boolean combinations of $\boldsymbol{\Pi}_{2}^{0}$-sets, hence $\boldsymbol{\Delta}_{3}^{0}$-sets Sta97, PP04.

The question of the topological complexity of relations on infinite words also naturally arises and is asked by Simonnet in Sim92. It is also posed in a more general form by Lescow and Thomas in LT94 (for infinite labelled partial orders) and in Tho88 where Thomas suggested to study reducibility notions and associated completeness results.

Every infinitary rational relation is an analytic set. We showed in [Fin03b that there exist some infinitary rational relations which are analytic but non Borel. Partial results about the Borel hierarchy of infinitary rational relations were first obtained in Fin03a, Fin07, where some $\boldsymbol{\Sigma}_{3}^{0}$-complete and some $\boldsymbol{\Pi}_{3}^{0}$-complete infinitary rational relations were constructed. In a recent conference paper we proved that for every non null recursive ordinal $\alpha$, there exist some $\boldsymbol{\Sigma}_{\alpha}^{0}$-complete and some $\boldsymbol{\Pi}_{\alpha}^{0}$-complete infinitary rational relations, Fin06b.

On the other hand, William W. (Bill) Wadge studied in Wad83 a great refinement of the Borel hierarchy defined via the notion of reduction by continuous functions. He determined the length of the new hierarchy obtained in that way, which is now called the Wadge hierarchy and has been much studied in descriptive set theory, see for example $\operatorname{Mos} 80$, Kec95. Dup01]. It is then natural to ask for the Wadge hierarchy of classes of $\omega$-languages accepted by finite machines, like Büchi automata or 2-tape Büchi automata. The Wagner hierarchy, effectively determined by Wagner, is actually, as noticed by Simonnet in [Sim92], the Wadge hierarchy of regular $\omega$-languages; its length is the ordinal $\omega^{\omega}$ [Wag79, Sel95, Sel98]. The Wadge hierarchy of $\omega$-languages accepted by Muller deterministic one blind (i. e. without zero-test) counter automata is an effective extension of the Wagner hierarchy studied in Fin01. Wadge degrees of deterministic context free $\omega$-languages have been determined by Duparc. The length of the Wadge hierarchy of deterministic context free $\omega$-languages is the ordinal $\omega^{\left(\omega^{2}\right)}$ DFR01, Dup03. Selivanov has recently determined the Wadge hierarchy of $\omega$-languages accepted by deterministic Turing machines; its length is $\left(\omega_{1}^{\mathrm{CK}}\right)^{\omega}$ Sel03a, Sel03b. 
On the other hand, we recently proved in [Fin06a that the Wadge hierarchy of $\omega$-languages accepted by non deterministic real time Büchi 1-counter automata is equal to the Wadge hierarchy of $\omega$-languages accepted by non deterministic Büchi Turing machines. Using a simulation of real time 1-counter automata, we show that, from a topological point of view, 2-tape Büchi automata have the same accepting power than Turing machines equipped with a Büchi acceptance condition. The Borel and the Wadge hierarchies of the class $\mathbf{R} \mathbf{A} \mathbf{T}_{\omega}$ of infinitary rational relations accepted by 2-tape Büchi automata are equal to the Borel and the Wadge hierarchies of $\omega$-languages accepted by real-time Büchi 1-counter automata or by Büchi Turing machines. In particular, for every non null recursive ordinal $\alpha$, there exist some $\boldsymbol{\Sigma}_{\alpha}^{0}$-complete and some $\boldsymbol{\Pi}_{\alpha}^{0}$-complete infinitary rational relations. And the supremum of the set of Borel ranks of infinitary rational relations is an ordinal $\gamma_{2}^{1}$ which is strictly greater than the first non recursive ordinal $\omega_{1}^{\mathrm{CK}}$. This very surprising result gives answers to questions of Simonnet [Sim92] and of Lescow and Thomas [Tho90, [T94].

This paper is an extended version of a conference paper which appeared in the Proceedings of the 23rd International Symposium on Theoretical Aspects of Computer Science, STACS 2006, Fin06b.

The paper is organized as follows. In section 2 we recall the notion of 2-tape automata and of real time 1-counter automata with Büchi acceptance condition. In section 3 we recall some definitions and properties of Borel and Wadge hierarchies, and we prove our main result in section 4 .

\section{2-tape automata and 1-counter automata}

We assume the reader to be familiar with the theory of formal $(\omega$-)languages Tho90, Sta97. We shall use usual notations of formal language theory.

When $\Sigma$ is a finite alphabet, a non-empty finite word over $\Sigma$ is any sequence $x=a_{1} \ldots a_{k}$, where $a_{i} \in \Sigma$ for $i=1, \ldots, k$, and $k$ is an integer $\geq 1$. The length of $x$ is $k$, denoted by $|x|$. The empty word has no letter and is denoted by $\lambda$; its length is 0 . For $x=a_{1} \ldots a_{k}$, we write $x(i)=a_{i}$ and $x[i]=x(1) \ldots x(i)$ for $i \leq k$ and $x[0]=\lambda . \Sigma^{\star}$ is the set of finite words (including the empty word) over $\Sigma$.

The first infinite ordinal is $\omega$. An $\omega$-word over $\Sigma$ is an $\omega$-sequence $a_{1} \ldots a_{n} \ldots$, where for all integers $i \geq 1, a_{i} \in \Sigma$. When $\sigma$ is an $\omega$-word over $\Sigma$, we write $\sigma=\sigma(1) \sigma(2) \ldots \sigma(n) \ldots$, where for all $i, \sigma(i) \in \Sigma$, and $\sigma[n]=\sigma(1) \sigma(2) \ldots \sigma(n)$ for all $n \geq 1$ and $\sigma[0]=\lambda$.

The set of $\omega$-words over the alphabet $\Sigma$ is denoted by $\Sigma^{\omega}$. An $\omega$-language over an alphabet $\Sigma$ is a subset of $\Sigma^{\omega}$. The complement (in $\Sigma^{\omega}$ ) of an $\omega$-language $V \subseteq \Sigma^{\omega}$ is $\Sigma^{\omega}-V$, denoted $V^{-}$. 
Infinitary rational relations are subsets of $\Sigma^{\omega} \times \Gamma^{\omega}$, where $\Sigma$ and $\Gamma$ are finite alphabets, which are accepted by 2-tape Büchi automata (2-BA).

Definition 2.1. A 2-tape Büchi automaton is a sextuple $\mathcal{T}=\left(K, \Sigma, \Gamma, \Delta, q_{0}, F\right)$, where $K$ is a finite set of states, $\Sigma$ and $\Gamma$ are finite alphabets, $\Delta$ is a finite subset of $K \times \Sigma^{\star} \times \Gamma^{\star} \times K$ called the set of transitions, $q_{0}$ is the initial state, and $F \subseteq K$ is the set of accepting states.

A computation $\mathcal{C}$ of the 2-tape Büchi automaton $\mathcal{T}$ is an infinite sequence of transitions

$$
\left(q_{0}, u_{1}, v_{1}, q_{1}\right),\left(q_{1}, u_{2}, v_{2}, q_{2}\right), \ldots,\left(q_{i-1}, u_{i}, v_{i}, q_{i}\right),\left(q_{i}, u_{i+1}, v_{i+1}, q_{i+1}\right), \ldots
$$

The computation is said to be successful iff there exists a final state $q_{f} \in F$ and infinitely many integers $i \geq 0$ such that $q_{i}=q_{f}$.

The input word of the computation is $u=u_{1} \cdot u_{2} \cdot u_{3} \ldots$

The output word of the computation is $v=v_{1} \cdot v_{2} \cdot v_{3} \ldots$

Then the input and the output words may be finite or infinite.

The infinitary rational relation $R(\mathcal{T}) \subseteq \Sigma^{\omega} \times \Gamma^{\omega}$ accepted by the 2-tape Büchi automaton $\mathcal{T}$ is the set of pairs $(u, v) \in \Sigma^{\omega} \times \Gamma^{\omega}$ such that $u$ and $v$ are the input and the output words of some successful computation $\mathcal{C}$ of $\mathcal{T}$.

The set of infinitary rational relations will be denoted by $\mathbf{R} \mathbf{A} \mathbf{T}_{\omega}$.

Definition 2.2. A (real time) 1-counter machine is a 4-tuple $\mathcal{M}=\left(K, \Sigma, \Delta, q_{0}\right)$, where $K$ is a finite set of states, $\Sigma$ is a finite input alphabet, $q_{0} \in K$ is the initial state, and the transition relation $\Delta$ is a subset of $K \times \Sigma \times\{0,1\} \times K \times\{0,1,-1\}$. If the machine $\mathcal{M}$ is in a state $q$ and $c \in \mathbb{N}$ is the content of the counter then the configuration (or global state) of $\mathcal{M}$ is $(q, c)$.

For $a \in \Sigma, q, q^{\prime} \in K$ and $c \in \mathbb{N}$, if $\left(q, a, i, q^{\prime}, j\right) \in \Delta$, where $i=0$ if $c=0$ and $i=1$ if $c \neq 0$, then we write:

$$
a:(q, c) \mapsto \mathcal{M}\left(q^{\prime}, c+j\right)
$$

Thus we see that the transition relation must satisfy:

if $\left(q, a, i, q^{\prime}, j\right) \in \Delta$ and $i=0$ then $j=0$ or $j=1$ (but $j$ may not be equal to -1 ).

Let $\sigma=a_{1} a_{2} \ldots a_{n}$ be a finite word over $\Sigma$. A sequence of configurations $r=$ $\left(q_{i}, c_{i}\right)_{1 \leq i \leq n+1}$ is called a run of $\mathcal{M}$ on $\sigma$, starting in configuration $(p, c)$, iff:

(1) $\left(q_{1}, c_{1}\right)=(p, c)$

(2) for each $i \in[1, n], a_{i}:\left(q_{i}, c_{i}\right) \mapsto \mathcal{M}\left(q_{i+1}, c_{i+1}\right)$

Let $\sigma=a_{1} a_{2} \ldots a_{n} \ldots$ be an $\omega$-word over $\Sigma$. An $\omega$-sequence of configurations $r=\left(q_{i}, c_{i}\right)_{i \geq 1}$ is called a run of $\mathcal{M}$ on $\sigma$, starting in configuration $(p, c)$, iff:

(1) $\left(q_{1}, c_{1}\right)=(p, c)$

(2) for each $i \geq 1, a_{i}:\left(q_{i}, c_{i}\right) \mapsto \mathcal{M}\left(q_{i+1}, c_{i+1}\right)$

For every such run, $\operatorname{In}(r)$ is the set of all states entered infinitely often during the run $r$. 
A run $r$ of $M$ on $\sigma$, starting in configuration $\left(q_{0}, 0\right)$, will be simply called " $a$ run of $M$ on $\sigma "$.

Definition 2.3. A (real time) Büchi 1-counter automaton is a 5-tuple

$$
\mathcal{M}=\left(K, \Sigma, \Delta, q_{0}, F\right) \text {, }
$$

where $\mathcal{M}^{\prime}=\left(K, \Sigma, \Delta, q_{0}\right)$ is a (real time) 1-counter machine and $F \subseteq K$ is the set of accepting states. The $\omega$-language accepted by $\mathcal{M}$ is

$$
L(\mathcal{M})=\left\{\sigma \in \Sigma^{\omega} \mid \text { there exists a run } r \text { of } \mathcal{M} \text { on } \sigma \text { such that } \operatorname{In}(r) \cap F \neq \emptyset\right\}
$$

The class of $\omega$-languages accepted by real time Büchi 1-counter automata will be denoted $\mathbf{r}-\mathbf{B C L}(1)_{\omega}$.

\section{Topology}

\subsection{Borel hierarchy and analytic sets}

We assume the reader to be familiar with basic notions of topology which may be found in [Mos80, LT94, Kec95, Sta97, PP04. There is a natural metric on the set $\Sigma^{\omega}$ of infinite words over a finite alphabet $\Sigma$ containing at least two letters which is called the prefix metric and defined as follows. For $u, v \in \Sigma^{\omega}$ and $u \neq v$ let $\delta(u, v)=2^{-l_{\operatorname{pref}(u, v)}}$ where $l_{\operatorname{pref}(u, v)}$ is the first integer $n$ such that the $(n+1)^{s t}$ letter of $u$ is different from the $(n+1)^{s t}$ letter of $v$. This metric induces on $\Sigma^{\omega}$ the usual Cantor topology for which open subsets of $\Sigma^{\omega}$ are in the form $W . \Sigma^{\omega}$, where $W \subseteq \Sigma^{\star}$. A set $L \subseteq \Sigma^{\omega}$ is a closed set iff its complement $\Sigma^{\omega}-L$ is an open set. Define now the Borel Hierarchy of subsets of $\Sigma^{\omega}$ :

Definition 3.1. For a non-null countable ordinal $\alpha$, the classes $\boldsymbol{\Sigma}_{\alpha}^{0}$ and $\boldsymbol{\Pi}_{\alpha}^{0}$ of the Borel Hierarchy on the topological space $\Sigma^{\omega}$ are defined as follows:

$\boldsymbol{\Sigma}_{1}^{0}$ is the class of open subsets of $\Sigma^{\omega}, \mathbf{\Pi}_{1}^{0}$ is the class of closed subsets of $\Sigma^{\omega}$, and for any countable ordinal $\alpha \geq 2$ :

$\boldsymbol{\Sigma}_{\alpha}^{0}$ is the class of countable unions of subsets of $\Sigma^{\omega}$ in $\bigcup_{\gamma<\alpha} \boldsymbol{\Pi}_{\gamma}^{0}$.

$\boldsymbol{\Pi}_{\alpha}^{0}$ is the class of countable intersections of subsets of $\Sigma^{\omega}$ in $\bigcup_{\gamma<\alpha} \boldsymbol{\Sigma}_{\gamma}^{0}$.

Recall some basic results about these classes:

\section{Proposition 3.2.}

(a) $\boldsymbol{\Sigma}_{\alpha}^{0} \cup \boldsymbol{\Pi}_{\alpha}^{0} \subsetneq \boldsymbol{\Sigma}_{\alpha+1}^{0} \cap \boldsymbol{\Pi}_{\alpha+1}^{0}$, for each countable ordinal $\alpha \geq 1$.

(b) $\cup_{\gamma<\alpha} \boldsymbol{\Sigma}_{\gamma}^{0}=\cup_{\gamma<\alpha} \boldsymbol{\Pi}_{\gamma}^{0} \subsetneq \boldsymbol{\Sigma}_{\alpha}^{0} \cap \boldsymbol{\Pi}_{\alpha}^{0}$, for each countable limit ordinal $\alpha$.

(c) A set $W \subseteq X^{\omega}$ is in the class $\boldsymbol{\Sigma}_{\alpha}^{0}$ iff its complement is in the class $\boldsymbol{\Pi}_{\alpha}^{0}$.

(d) $\boldsymbol{\Sigma}_{\alpha}^{0}-\boldsymbol{\Pi}_{\alpha}^{0} \neq \emptyset$ and $\boldsymbol{\Pi}_{\alpha}^{0}-\boldsymbol{\Sigma}_{\alpha}^{0} \neq \emptyset$ hold for every countable ordinal $\alpha \geq 1$.

For a countable ordinal $\alpha$, a subset of $\Sigma^{\omega}$ is a Borel set of $\operatorname{rank} \alpha$ iff it is in $\boldsymbol{\Sigma}_{\alpha}^{0} \cup \boldsymbol{\Pi}_{\alpha}^{0}$ but not in $\bigcup_{\gamma<\alpha}\left(\Sigma_{\gamma}^{0} \cup \boldsymbol{\Pi}_{\gamma}^{0}\right)$.

There are also some subsets of $\Sigma^{\omega}$ which are not Borel. Indeed there exists another hierarchy beyond the Borel hierarchy, which is called the projective hierarchy and 
which is obtained from the Borel hierarchy by successive applications of operations of projection and complementation. The first level of the projective hierarchy is formed by the class of analytic sets and the class of co-analytic sets which are complements of analytic sets. In particular the class of Borel subsets of $\Sigma^{\omega}$ is strictly included into the class $\boldsymbol{\Sigma}_{1}^{1}$ of analytic sets which are obtained by projection of Borel sets.

Definition 3.3. A subset $A$ of $\Sigma^{\omega}$ is in the class $\Sigma_{1}^{1}$ of analytic sets iff there exists another finite set $Y$ and a Borel subset $B$ of $(\Sigma \times Y)^{\omega}$ such that $x \in A \leftrightarrow \exists y \in Y^{\omega}$ such that $(x, y) \in B$, where $(x, y)$ is the infinite word over the alphabet $\Sigma \times Y$ such that $(x, y)(i)=(x(i), y(i))$ for each integer $i \geq 1$.

We now define completeness with regard to reduction by continuous functions. For a countable ordinal $\alpha \geq 1$, a set $F \subseteq \Sigma^{\omega}$ is said to be a $\boldsymbol{\Sigma}_{\alpha}^{0}$ (respectively, $\boldsymbol{\Pi}_{\alpha}^{0}, \boldsymbol{\Sigma}_{1}^{1}$ )-complete set iff for any set $E \subseteq Y^{\omega}$ (with $Y$ a finite alphabet): $E \in \boldsymbol{\Sigma}_{\alpha}^{0}$ (respectively, $E \in \boldsymbol{\Pi}_{\alpha}^{0}, E \in \boldsymbol{\Sigma}_{1}^{1}$ ) iff there exists a continuous function $f: Y^{\omega} \rightarrow \Sigma^{\omega}$ such that $E=f^{-1}(F) . \boldsymbol{\Sigma}_{n}^{0}$ (respectively $\boldsymbol{\Pi}_{n}^{0}$ )-complete sets, with $n$ an integer $\geq 1$, are thoroughly characterized in Sta86.

In particular $\mathcal{R}=\left(0^{\star} .1\right)^{\omega}$ is a well known example of $\boldsymbol{\Pi}_{2}^{0}$-complete subset of $\{0,1\}^{\omega}$. It is the set of $\omega$-words over $\{0,1\}$ having infinitely many occurrences of the letter 1 . Its complement $\{0,1\}^{\omega}-\left(0^{\star} .1\right)^{\omega}$ is a $\boldsymbol{\Sigma}_{2}^{0}$-complete subset of $\{0,1\}^{\omega}$.

We recall now the definition of the arithmetical hierarchy of $\omega$-languages which form the effective analogue to the hierarchy of Borel sets of finite ranks.

Let $X$ be a finite alphabet. An $\omega$-language $L \subseteq X^{\omega}$ belongs to the class $\Sigma_{n}$ if and only if there exists a recursive relation $R_{L} \subseteq(\mathbb{N})^{n-1} \times X^{\star}$ such that

$$
L=\left\{\sigma \in X^{\omega} \mid \exists a_{1} \ldots Q_{n} a_{n} \quad\left(a_{1}, \ldots, a_{n-1}, \sigma\left[a_{n}+1\right]\right) \in R_{L}\right\}
$$

where $Q_{i}$ is one of the quantifiers $\forall$ or $\exists$ (not necessarily in an alternating order). An $\omega$-language $L \subseteq X^{\omega}$ belongs to the class $\Pi_{n}$ if and only if its complement $X^{\omega}-L$ belongs to the class $\Sigma_{n}$. The inclusion relations that hold between the classes $\Sigma_{n}$ and $\Pi_{n}$ are the same as for the corresponding classes of the Borel hierarchy. The classes $\Sigma_{n}$ and $\Pi_{n}$ are included in the respective classes $\boldsymbol{\Sigma}_{\mathbf{n}}^{\mathbf{0}}$ and $\boldsymbol{\Sigma}_{\mathbf{n}}^{\mathbf{0}}$ of the Borel hierarchy, and cardinality arguments suffice to show that these inclusions are strict.

As in the case of the Borel hierarchy, projections of arithmetical sets (of the second $\Pi$-class) lead beyond the arithmetical hierarchy, to the analytical hierarchy of $\omega$ languages. The first class of this hierarchy is the (lightface) class $\Sigma_{1}^{1}$ of effective analytic sets which are obtained by projection of arithmetical sets. The (lightface) class $\Pi_{1}^{1}$ of effective co-analytic sets is simply the class of complements of effective analytic sets. We denote as usual $\Delta_{1}^{1}=\Sigma_{1}^{1} \cap \Pi_{1}^{1}$. 
It is well known that an $\omega$-language $L \subseteq X^{\omega}$ is in the class $\Sigma_{1}^{1}$ iff it is accepted by a non deterministic Turing machine with a Büchi or Muller acceptance condition Sta97.

Borel ranks of (lightface) $\Delta_{1}^{1}$ sets are the (recursive) ordinals $\gamma<\omega_{1}^{\mathrm{CK}}$, where $\omega_{1}^{\mathrm{CK}}$ is the first non-recursive ordinal, usually called the Church-Kleene ordinal. Moreover, for every non null ordinal $\alpha<\omega_{1}^{\mathrm{CK}}$, there exist some $\boldsymbol{\Sigma}_{\alpha}^{0}$-complete and some $\boldsymbol{\Pi}_{\alpha}^{0}$-complete sets in the class $\Delta_{1}^{1}$. On the other hand, Kechris, Marker and Sami proved in KMS89] that the supremum of the set of Borel ranks of (lightface) $\Sigma_{1}^{1}$-sets is an ordinal $\gamma_{2}^{1}$ which is strictly greater than the ordinal $\delta_{2}^{1}$ which is the first non $\Delta_{2}^{1}$ ordinal. Thus the ordinal $\gamma_{2}^{1}$ is also strictly greater than the ChurchKleene ordinal $\omega_{1}^{\mathrm{CK}}$. The exact value of the ordinal $\gamma_{2}^{1}$ may depend on axioms of set theory KMS89. It is consistent with the axiomatic system ZFC that $\gamma_{2}^{1}$ is equal to the ordinal $\delta_{3}^{1}$ which is the first non $\Delta_{3}^{1}$ ordinal (because $\gamma_{2}^{1}=\delta_{3}^{1}$ in ZFC $+(\mathbf{V}=\mathbf{L}))$. On the other hand the axiom of $\Pi_{1}^{1}$-determinacy implies that $\gamma_{2}^{1}<\delta_{3}^{1}$. For more details, we refer the reader to [KMS89] and to a textbook of set theory like Jec02.

Notice however that it seems still unknown whether every non null ordinal $\gamma<\gamma_{2}^{1}$ is the Borel rank of a (lightface) $\Sigma_{1}^{1}$-set.

\subsection{Wadge hierarchy}

We now introduce the Wadge hierarchy, which is a great refinement of the Borel hierarchy defined via reductions by continuous functions, Wad83, Dup01.

Definition 3.4 (Wadge Wad83). Let $X, Y$ be two finite alphabets. For $L \subseteq X^{\omega}$ and $L^{\prime} \subseteq Y^{\omega}, L$ is said to be Wadge reducible to $L^{\prime}\left(L \leq_{W} L^{\prime}\right)$ iff there exists a continuous function $f: X^{\omega} \rightarrow Y^{\omega}$, such that $L=f^{-1}\left(L^{\prime}\right)$.

$L$ and $L^{\prime}$ are Wadge equivalent iff $L \leq_{W} L^{\prime}$ and $L^{\prime} \leq_{W} L$. This will be denoted by $L \equiv_{W} L^{\prime}$. And we shall say that $L<_{W} L^{\prime}$ iff $L \leq_{W} L^{\prime}$ but not $L^{\prime} \leq_{W} L$.

A set $L \subseteq X^{\omega}$ is said to be self dual iff $L \equiv_{W} L^{-}$, and otherwise it is said to be non self dual.

The relation $\leq_{W}$ is reflexive and transitive, and $\equiv_{W}$ is an equivalence relation.

The equivalence classes of $\equiv_{W}$ are called Wadge degrees.

The Wadge hierarchy $W H$ is the class of Borel subsets of a set $X^{\omega}$, where $X$ is a finite set, equipped with $\leq_{W}$ and with $\equiv_{W}$.

For $L \subseteq X^{\omega}$ and $L^{\prime} \subseteq Y^{\omega}$, if $L \leq_{W} L^{\prime}$ and $L=f^{-1}\left(L^{\prime}\right)$ where $f$ is a continuous function from $X^{\omega}$ into $Y^{\omega}$, then $f$ is called a continuous reduction of $L$ to $L^{\prime}$. Intuitively it means that $L$ is less complicated than $L^{\prime}$ because to check whether $x \in L$ it suffices to check whether $f(x) \in L^{\prime}$ where $f$ is a continuous function. Hence the Wadge degree of an $\omega$-language is a measure of its topological complexity.

Notice that in the above definition, we consider that a subset $L \subseteq X^{\omega}$ is given together with the alphabet $X$. This is important as it is shown by the following simple example. Let $L_{1}=\{0,1\}^{\omega} \subseteq\{0,1\}^{\omega}$ and $L_{2}=\{0,1\}^{\omega} \subseteq\{0,1,2\}^{\omega}$. So 
the languages $L_{1}$ and $L_{2}$ are equal but considered over the different alphabets $X_{1}=\{0,1\}$ and $X_{2}=\{0,1,2\}$. It turns out that $L_{1}<_{W} L_{2}$. In fact $L_{1}$ is open and closed in $X_{1}^{\omega}$ while $L_{2}$ is closed but non open in $X_{2}^{\omega}$.

We can now define the Wadge class of a set $L$ :

Definition 3.5. Let $L$ be a subset of $X^{\omega}$. The Wadge class of $L$ is :

$$
[L]=\left\{L^{\prime} \mid L^{\prime} \subseteq Y^{\omega} \text { for a finite alphabet } Y \text { and } L^{\prime} \leq_{W} L\right\} .
$$

Recall that each Borel class $\boldsymbol{\Sigma}_{\alpha}^{0}$ and $\mathbf{\Pi}_{\alpha}^{0}$ is a Wadge class.

A set $L \subseteq X^{\omega}$ is a $\boldsymbol{\Sigma}_{\alpha}^{0}$ (respectively $\boldsymbol{\Pi}_{\alpha}^{0}$ )-complete set iff for any set $L^{\prime} \subseteq Y^{\omega}, L^{\prime}$ is in $\boldsymbol{\Sigma}_{\alpha}^{0}$ (respectively $\boldsymbol{\Pi}_{\alpha}^{0}$ ) iff $L^{\prime} \leq_{W} L$. It follows from the study of the Wadge hierarchy that a set $L \subseteq X^{\omega}$ is a $\boldsymbol{\Sigma}_{\alpha}^{0}$ (respectively, $\boldsymbol{\Pi}_{\alpha}^{0}$ )-complete set iff it is in $\boldsymbol{\Sigma}_{\alpha}^{0}$ but not in $\boldsymbol{\Pi}_{\alpha}^{0}$ (respectively, in $\boldsymbol{\Pi}_{\alpha}^{0}$ but not in $\boldsymbol{\Sigma}_{\alpha}^{0}$ ).

There is a close relationship between Wadge reducibility and games which we now introduce.

Definition 3.6 (Wadge Wad83). Let $L \subseteq X^{\omega}$ and $L^{\prime} \subseteq Y^{\omega}$. The Wadge game $W\left(L, L^{\prime}\right)$ is a game with perfect information between two players, player 1 who is in charge of $L$ and player 2 who is in charge of $L^{\prime}$.

Player 1 first writes a letter $a_{1} \in X$, then player 2 writes a letter $b_{1} \in Y$, then player 1 writes a letter $a_{2} \in X$, and so on.

The two players alternatively write letters $a_{n}$ of $X$ for player 1 and $b_{n}$ of $Y$ for player 2.

After $\omega$ steps, the player 1 has written an $\omega$-word $a \in X^{\omega}$ and the player 2 has written an $\omega$-word $b \in Y^{\omega}$. The player 2 is allowed to skip, even infinitely often, provided he really writes an $\omega$-word in $\omega$ steps.

The player 2 wins the play iff $\left[a \in L \leftrightarrow b \in L^{\prime}\right]$, i.e. iff :

[( $a \in L$ and $\left.b \in L^{\prime}\right)$ or ( $a \notin L$ and $b \notin L^{\prime}$ and $b$ is infinite)].

Recall that a strategy for player 1 is a function $\sigma:(Y \cup\{s\})^{\star} \rightarrow X$. And a strategy for player 2 is a function $f: X^{+} \rightarrow Y \cup\{s\}$.

$\sigma$ is a winning stategy for player 1 iff he always wins a play when he uses the strategy $\sigma$, i.e. when the $n^{\text {th }}$ letter he writes is given by $a_{n}=\sigma\left(b_{1} \ldots b_{n-1}\right)$, where $b_{i}$ is the letter written by player 2 at step $i$ and $b_{i}=s$ if player 2 skips at step $i$. A winning strategy for player 2 is defined in a similar manner.

Martin's Theorem states that every Gale-Stewart Game $G(X)$, with $X$ a Borel set, is determined, see Kec95. This implies the following determinacy result :

Theorem 3.7 (Wadge). Let $L \subseteq X^{\omega}$ and $L^{\prime} \subseteq Y^{\omega}$ be two Borel sets, where $X$ and $Y$ are finite alphabets. Then the Wadge game $W\left(L, L^{\prime}\right)$ is determined: one of the two players has a winning strategy. And $L \leq_{W} L^{\prime}$ iff the player 2 has a winning strategy in the game $W\left(L, L^{\prime}\right)$. 
Theorem 3.8 (Wadge). Up to the complement and $\equiv_{W}$, the class of Borel subsets of $X^{\omega}$, for a finite alphabet $X$, is a well ordered hierarchy. There is an ordinal $|W H|$, called the length of the hierarchy, and a map $d_{W}^{0}$ from $W H$ onto $|W H|-\{0\}$, such that for all $L, L^{\prime} \subseteq X^{\omega}$ :

$d_{W}^{0} L<d_{W}^{0} L^{\prime} \leftrightarrow \bar{L}<_{W} L^{\prime}$ and

$d_{W}^{0} L=d_{W}^{0} L^{\prime} \leftrightarrow\left[L \equiv_{W} L^{\prime}\right.$ or $\left.L \equiv_{W} L^{\prime-}\right]$.

The Wadge hierarchy of Borel sets of finite rank has length ${ }^{1} \varepsilon_{0}$ where ${ }^{1} \varepsilon_{0}$ is the limit of the ordinals $\alpha_{n}$ defined by $\alpha_{1}=\omega_{1}$ and $\alpha_{n+1}=\omega_{1}^{\alpha_{n}}$ for $n$ a non negative integer, $\omega_{1}$ being the first non countable ordinal. Then ${ }^{1} \varepsilon_{0}$ is the first fixed point of the ordinal exponentiation of base $\omega_{1}$. The length of the Wadge hierarchy of Borel sets in $\boldsymbol{\Delta}_{\omega}^{\mathbf{0}}=\boldsymbol{\Sigma}_{\omega}^{\mathbf{0}} \cap \boldsymbol{\Pi}_{\omega}^{\mathbf{0}}$ is the $\omega_{1}^{t h}$ fixed point of the ordinal exponentiation of base $\omega_{1}$, which is a much larger ordinal. The length of the whole Wadge hierarchy of Borel sets is a huge ordinal, with regard to the $\omega_{1}^{t h}$ fixed point of the ordinal exponentiation of base $\omega_{1}$. It has been determined by Wadge and is described in Wad83, Dup01 by the use of the Veblen functions.

\section{Wadge hierarchy of infinitary rational relations}

We have proved in Fin06a the following result.

Theorem 4.1 ([Fin06a $)$. The Wadge hierarchy of the class $\mathbf{r}-\mathbf{B C L}(1)_{\omega}$ is equal to the Wadge hierarchy of the class $\Sigma_{1}^{1}$ of $\omega$-languages accepted by Turing machines with a Büchi acceptance condition.

We are going to prove a similar result for the class $\mathbf{R} \mathbf{A} \mathbf{T}_{\omega}$, using a simulation of 1-counter automata.

Theorem 4.2. The Wadge hierarchy of the class $\mathbf{R A} \mathbf{T}_{\omega}$ is equal to the Wadge hierarchy of the class $\mathbf{r}-\mathbf{B C L}(1)_{\omega}$ hence also of the class $\Sigma_{1}^{1}$ of $\omega$-languages accepted by Turing machines with a Büchi acceptance condition.

We now first define a coding of an $\omega$-word over a finite alphabet $\Sigma$, such that $0 \in \Sigma$, by an $\omega$-word over the alphabet $\Gamma=\Sigma \cup\{A\}$, where $A$ is an additional letter not in $\Sigma$.

For $x \in \Sigma^{\omega}$ the $\omega$-word $h(x)$ is defined by:

$h(x)=A \cdot 0 \cdot x(1) \cdot A \cdot 0^{2} \cdot x(2) \cdot A \cdot 0^{3} \cdot x(3) \cdot A \cdot 0^{4} \cdot x(4) \cdot A \ldots \ldots \cdot 0^{n} \cdot x(n) \cdot A \cdot 0^{n+1} \cdot x(n+1) \cdot A \ldots$

Then it is easy to see that the mapping $h$ from $\Sigma^{\omega}$ into $(\Sigma \cup\{A\})^{\omega}$ is continuous and injective.

Let now $\alpha$ be the $\omega$-word over the alphabet $\Sigma \cup\{A\}$ which is defined by:

$$
\alpha=A \cdot 0 \cdot A \cdot 0^{2} \cdot A \cdot 0^{3} \cdot A \cdot 0^{4} \cdot A \cdot 0^{5} \cdot A \ldots \cdot A \cdot 0^{n} \cdot A \cdot 0^{n+1} \cdot A \ldots
$$

We can now state the following Lemma. 
Lemma 4.3. Let $\Sigma$ be a finite alphabet such that $0 \in \Sigma, \alpha$ be the $\omega$-word over $\Sigma \cup\{A\}$ defined as above, and $L \subseteq \Sigma^{\omega}$ be in $\mathbf{r}-\mathbf{B C L}(1)_{\omega}$. Then there exists an infinitary rational relation $R_{1} \subseteq(\Sigma \cup\{A\})^{\omega} \times(\Sigma \cup\{A\})^{\omega}$ such that:

$$
\forall x \in \Sigma^{\omega}(x \in L) \text { iff }\left((h(x), \alpha) \in R_{1}\right)
$$

Proof. Let $\Sigma$ be a finite alphabet such that $0 \in \Sigma, \alpha$ be the $\omega$-word over $\Sigma \cup\{A\}$ defined as above, and $L=L(\mathcal{A}) \subseteq \Sigma^{\omega}$, where $\mathcal{A}=\left(K, \Sigma, \Delta, q_{0}, F\right)$ is a real time 1-counter Büchi automaton.

We define now the relation $R_{1}$. A pair $y=\left(y_{1}, y_{2}\right)$ of $\omega$-words over the alphabet $\Sigma \cup\{A\}$ is in $R_{1}$ if and only if it is in the form

$y_{1}=A \cdot u_{1} \cdot v_{1} \cdot x(1) \cdot A \cdot u_{2} \cdot v_{2} \cdot x(2) \cdot A \cdot u_{3} \cdot v_{3} \cdot x(3) \cdot A \ldots A \cdot u_{n} \cdot v_{n} \cdot x(n) \cdot A \ldots$

$y_{2}=A \cdot w_{1} \cdot z_{1} \cdot A \cdot w_{2} \cdot z_{2} \cdot A \cdot w_{3} \cdot z_{3} \cdot A \ldots A \cdot w_{n} \cdot z_{n} \cdot A \ldots$

where $\left|v_{1}\right|=0$ and for all integers $i \geq 1$,

$$
\begin{gathered}
u_{i}, v_{i}, w_{i}, z_{i} \in 0^{\star} \text { and } x(i) \in \Sigma \text { and } \\
\left|u_{i+1}\right|=\left|z_{i}\right|+1
\end{gathered}
$$

and there is a sequence $\left(q_{i}\right)_{i \geq 0}$ of states of $K$ such that for all integers $i \geq 1$ :

$$
x(i):\left(q_{i-1},\left|v_{i}\right|\right) \mapsto_{\mathcal{A}}\left(q_{i},\left|w_{i}\right|\right)
$$

Moreover some state $q_{f} \in F$ occurs infinitely often in the sequence $\left(q_{i}\right)_{i \geq 0}$.

Notice that the state $q_{0}$ of the sequence $\left(q_{i}\right)_{i \geq 0}$ is also the initial state of $\mathcal{A}$.

Notice that the main idea is that we try to simulate, using a 2-tape automaton, the reading of the infinite word $x(1) . x(2) . x(3) \ldots$ by the real time 1-counter Büchi automaton $\mathcal{A}$. The initial value of the counter is $\left|v_{1}\right|$ and the value of the counter after the reading of the letter $x(1)$ by $\mathcal{A}$ is $\left|w_{1}\right|$ which is on the second tape. Now the 2-tape automaton accepting $R_{1}$ would need to read again the value $\left|w_{1}\right|$ in order to compare it to the value of the counter after the reading of $x(2)$ by the 1 -counter automaton $\mathcal{A}$. This is not directly possible so the simulation does not work on every pair of $R_{1}$. However, using the very special shape of pairs in $h\left(\Sigma^{\omega}\right) \times\{\alpha\}$, the simulation will be possible on a pair $(h(x), \alpha)$. Then for such a pair $(h(x), \alpha) \in R_{1}$ written in the above form $\left(y_{1}, y_{2}\right)$, we have $\left|v_{2}\right|=\left|w_{1}\right|$ and then the simulation can continue from the value $\left|v_{2}\right|$ of the counter, and so on.

We now give the details of the proof.

Let $x \in \Sigma^{\omega}$ be such that $(h(x), \alpha) \in R_{1}$. We are going to prove that $x \in L$.

By hypothesis $(h(x), \alpha) \in R_{1}$ thus there are finite words $u_{i}, v_{i}, w_{i}, z_{i} \in 0^{\star}$ such that $\left|v_{1}\right|=0$ and for all integers $i \geq 1,\left|u_{i+1}\right|=\left|z_{i}\right|+1$, and

$$
h(x)=A \cdot u_{1} \cdot v_{1} \cdot x(1) \cdot A \cdot u_{2} \cdot v_{2} \cdot x(2) \cdot A \cdot u_{3} \cdot v_{3} \cdot x(3) \cdot A \ldots A \cdot u_{n} \cdot v_{n} \cdot x(n) \cdot A \ldots
$$


$\alpha=A \cdot w_{1} \cdot z_{1} \cdot A \cdot w_{2} \cdot z_{2} \cdot A \cdot w_{3} \cdot z_{3} \cdot A \ldots A \cdot w_{n} \cdot z_{n} \cdot A \ldots$

Moreover there is a sequence $\left(q_{i}\right)_{i \geq 0}$ of states of $K$ such that for all integers $i \geq 1$ :

$$
x(i):\left(q_{i-1},\left|v_{i}\right|\right) \mapsto_{\mathcal{A}}\left(q_{i},\left|w_{i}\right|\right)
$$

and some state $q_{f} \in F$ occurs infinitely often in the sequence $\left(q_{i}\right)_{i \geq 0}$.

On the other side we have:

$h(x)=A \cdot 0 \cdot x(1) \cdot A \cdot 0^{2} \cdot x(2) \cdot A \cdot 0^{3} \cdot x(3) \cdot A \ldots A \cdot 0^{n} \cdot x(n) \cdot A \cdot 0^{n+1} \cdot x(n+1) \cdot A \ldots$

$\alpha=A \cdot 0 \cdot A \cdot 0^{2} \cdot A \cdot 0^{3} \cdot A \cdot 0^{4} \cdot A \ldots A \cdot 0^{n} \cdot A \ldots$

So we have $\left|u_{1} \cdot v_{1}\right|=1$ and $\left|v_{1}\right|=0$ and $x(1):\left(q_{0},\left|v_{1}\right|\right) \mapsto_{\mathcal{A}}\left(q_{1},\left|w_{1}\right|\right)$. But $\left|w_{1} \cdot z_{1}\right|=1,\left|u_{2} \cdot v_{2}\right|=2$, and $\left|u_{2}\right|=\left|z_{1}\right|+1$ thus $\left|v_{2}\right|=\left|w_{1}\right|$.

We are going to prove in a similar way that for all integers $i \geq 1$ it holds that $\left|v_{i+1}\right|=\left|w_{i}\right|$.

We know that $\left|w_{i} . z_{i}\right|=i,\left|u_{i+1} \cdot v_{i+1}\right|=i+1$, and $\left|u_{i+1}\right|=\left|z_{i}\right|+1$ thus $\left|w_{i}\right|=\left|v_{i+1}\right|$.

Then for all $i \geq 1, x(i):\left(q_{i-1},\left|v_{i}\right|\right) \mapsto \mathcal{A}\left(q_{i},\left|v_{i+1}\right|\right)$.

So if we set $c_{i}=\left|v_{i}\right|,\left(q_{i-1}, c_{i}\right)_{i \geq 1}$ is an accepting run of $\mathcal{A}$ on $x$ and this implies that $x \in L$.

Conversely it is easy to prove that if $x \in L$ then $(h(x), \alpha)$ may be written in the form of $\left(y_{1}, y_{2}\right) \in R_{1}$.

It remains to prove that the above defined relation $R_{1}$ is an infinitary rational relation. It is easy to find a 2 -tape Büchi automaton $\mathcal{T}$ accepting the relation $R_{1}$.

Lemma 4.4. The set

$$
R_{2}=(\Sigma \cup\{A\})^{\omega} \times(\Sigma \cup\{A\})^{\omega}-\left(h\left(\Sigma^{\omega}\right) \times\{\alpha\}\right)
$$

is an infinitary rational relation.

Proof. By definition of the mapping $h$, we know that a pair of $\omega$-words over the alphabet $(\Sigma \cup\{A\})$ is in $h\left(\Sigma^{\omega}\right) \times\{\alpha\}$ iff it is in the form $\left(\sigma_{1}, \sigma_{2}\right)$, where $\sigma_{1}=A \cdot 0 \cdot x(1) \cdot A \cdot 0^{2} \cdot x(2) \cdot A \cdot 0^{3} \cdot x(3) \cdot A \ldots \cdot A \cdot 0^{n} \cdot x(n) \cdot A \cdot 0^{n+1} \cdot x(n+1) \cdot A \ldots$ $\sigma_{2}=\alpha=A \cdot 0 \cdot A \cdot 0^{2} \cdot A \cdot 0^{3} \cdot A \ldots A \cdot 0^{n} \cdot A \cdot 0^{n+1} \cdot A \ldots$

where for all integers $i \geq 1, x(i) \in \Sigma$.

So it is easy to see that $(\Sigma \cup\{A\})^{\omega} \times(\Sigma \cup\{A\})^{\omega}-\left(h\left(\Sigma^{\omega}\right) \times\{\alpha\}\right)$ is the union of the sets $\mathcal{C}_{j}$ where: 
- $\mathcal{C}_{1}$ is formed by pairs $\left(\sigma_{1}, \sigma_{2}\right)$ where $\sigma_{1}$ has not any initial segment in $A \cdot \Sigma^{2} \cdot A \cdot \Sigma^{3} \cdot A$, or $\sigma_{2}$ has not any initial segment in $A . \Sigma . A . \Sigma^{2}$.A.

- $\mathcal{C}_{2}$ is formed by pairs $\left(\sigma_{1}, \sigma_{2}\right)$ where $\sigma_{2} \notin\left(A .0^{+}\right)^{\omega}$, or $\sigma_{1} \notin\left(A .0^{+} . \Sigma\right)^{\omega}$.

- $\mathcal{C}_{3}$ is formed by pairs $\left(\sigma_{1}, \sigma_{2}\right)$ where $\sigma_{1}=A \cdot w_{1} \cdot A \cdot w_{2} \cdot A \cdot w_{3} \cdot A \ldots \cdot A \cdot w_{n} \cdot A \cdot u \cdot A \cdot z_{1}$ $\sigma_{2}=A \cdot w_{1}^{\prime} \cdot A \cdot w_{2}^{\prime} \cdot A \cdot w_{3}^{\prime} \cdot A \ldots \cdot A \cdot w_{n}^{\prime} \cdot A \cdot v \cdot A \cdot z_{2}$

where $n$ is an integer $\geq 1$, for all $i \leq n w_{i}, w_{i}^{\prime} \in \Sigma^{\star}, z_{1}, z_{2} \in(\Sigma \cup\{A\})^{\omega}$ and

$$
u, v \in \Sigma^{\star} \text { and }|u| \neq|v|+1
$$

- $\mathcal{C}_{4}$ is formed by pairs $\left(\sigma_{1}, \sigma_{2}\right)$ where $\sigma_{1}=A \cdot w_{1} \cdot A \cdot w_{2} \cdot A \cdot w_{3} \cdot A \cdot w_{4} \ldots \cdot A \cdot w_{n} \cdot A \cdot w_{n+1} \cdot A \cdot v \cdot A \cdot z_{1}$ $\sigma_{2}=A \cdot w_{1}^{\prime} \cdot A \cdot w_{2}^{\prime} \cdot A \cdot w_{3}^{\prime} \cdot A \cdot w_{4}^{\prime} \ldots A \cdot w_{n}^{\prime} \cdot A \cdot u \cdot A \cdot z_{2}$

where $n$ is an integer $\geq 1$, for all $i \leq n w_{i}, w_{i}^{\prime} \in \Sigma^{\star}, w_{n+1} \in \Sigma^{\star}, z_{1}, z_{2} \in$ $(\Sigma \cup\{A\})^{\omega}$ and

$$
u, v \in \Sigma^{\star} \text { and }|v| \neq|u|+2
$$

Each set $\mathcal{C}_{j}, 1 \leq j \leq 4$, is easily seen to be an infinitary rational relation $\subseteq$ $(\Sigma \cup\{A\})^{\omega} \times(\Sigma \cup\{A\})^{\omega}$ (the detailed proof is left to the reader). The class $\mathbf{R A T}_{\omega}$ is closed under finite union thus

$$
R_{2}=(\Sigma \cup\{A\})^{\omega} \times(\Sigma \cup\{A\})^{\omega}-\left(h\left(\Sigma^{\omega}\right) \times\{\alpha\}\right)=\bigcup_{1 \leq j \leq 4} \mathcal{C}_{j}
$$

is an infinitary rational relation.

As in Fin06a, we are going to consider first non self dual sets to prove Theorem 4.2. We recall the definition of Wadge degrees introduced by Duparc in Dup01 and which is a slight modification of the previous one.

\section{Definition 4.5.}

(a) $d_{w}(\emptyset)=d_{w}\left(\emptyset^{-}\right)=1$

(b) $d_{w}(L)=\sup \left\{d_{w}\left(L^{\prime}\right)+1 \mid L^{\prime}\right.$ non self dual and $\left.L^{\prime}<_{W} L\right\}$ (for either $L$ self dual or not, $L>_{W} \emptyset$ ).

We are going now to introduce the operation of sum of sets of infinite words which has as counterpart the ordinal addition over Wadge degrees.

Definition 4.6 (Wadge, see Wad83, Dup01]). Assume that $X \subseteq Y$ are two finite alphabets, $Y-X$ containing at least two elements, and that $\left\{X_{+}, X_{-}\right\}$is a partition of $Y-X$ in two non empty sets. Let $L \subseteq X^{\omega}$ and $L^{\prime} \subseteq Y^{\omega}$, then

$L^{\prime}+L={ }_{d f} L \cup\left\{u . a . \beta \mid u \in X^{\star},\left(a \in X_{+}\right.\right.$and $\left.\beta \in L^{\prime}\right)$ or $\left(a \in X_{-}\right.$and $\left.\left.\beta \in L^{\prime-}\right)\right\}$

This operation is closely related to the ordinal sum as it is stated in the following: 
Theorem 4.7 (Wadge, see Wad83, Dup01). Let $X \subseteq Y, Y-X$ containing at least two elements, $L \subseteq X^{\omega}$ and $L^{\prime} \subseteq Y^{\omega}$ be non self dual Borel sets. Then $\left(L+L^{\prime}\right)$ is a non self dual Borel set and $d_{w}\left(L^{\prime}+L\right)=d_{w}\left(L^{\prime}\right)+d_{w}(L)$.

A player in charge of a set $L^{\prime}+L$ in a Wadge game is like a player in charge of the set $L$ but who can, at any step of the play, erase his previous play and choose to be this time in charge of $L^{\prime}$ or of $L^{\prime-}$. Notice that he can do this only one time during a play. The following lemma was proved in Fin06a.

Lemma 4.8. Let $L \subseteq \Sigma^{\omega}$ be a non self dual Borel set such that $d_{w}(L) \geq \omega$. Then it holds that $L \equiv_{W} \bar{\emptyset}+L$.

Notice that in the above lemma, $\emptyset$ is viewed as the empty set over an alphabet $\Sigma^{\prime}$ such that $\Sigma \subseteq \Sigma^{\prime}$ and cardinal $\left(\Sigma^{\prime}-\Sigma\right) \geq 2$.

Proof. Assume that $L \subseteq \Sigma^{\omega}$ is a non self dual Borel set and that $d_{w}(L) \geq \omega$. We know that $\emptyset$ is a non self dual Borel set and that $d_{w}(\emptyset)=1$. Thus, by Theorem 4.7, it holds that $d_{w}(\emptyset+L)=d_{w}(\emptyset)+d_{w}(L)=1+d_{w}(L)$. But by hypothesis $d_{w}(L) \geq \omega$ and this implies that $1+d_{w}(L)=d_{w}(L)$. So we have proved that $d_{w}(\emptyset+L)=d_{w}(L)$.

On the other hand $L$ is non self dual and $d_{w}(\emptyset+L)=d_{w}(L)$ imply that only two cases may happen : either $\emptyset+L \equiv_{W} L$ or $\emptyset+L \equiv_{W} L^{-}$.

But it is easy to see that $L \leq_{W} \emptyset+L$. For that purpose consider the Wadge game $W(L, \emptyset+L)$. Player 2 has clearly a winning strategy which consists in copying the play of Player 1 thus $L \leq_{W} \emptyset+L$. This implies that $\emptyset+L \equiv_{W} L^{-}$cannot hold so $\emptyset+L \equiv_{W} L$.

We can now state the following lemma.

Lemma 4.9. Let $L \subseteq \Sigma^{\omega}$ be a non self dual Borel set accepted by a real time Büchi 1-counter automaton. Then there is an infinitary rational relation $R \subseteq$ $(\Sigma \cup\{A\})^{\omega} \times(\Sigma \cup\{A\})^{\omega}$ such that $L \equiv_{W} R$.

Proof. It is well known that there are regular $\omega$-languages of every finite Wadge degree, Sta97, Sel98. These $\omega$-languages are Boolean combinations of open sets. So we have only to consider the case of non self dual Borel sets of Wadge degrees greater than or equal to $\omega$.

Let then $L=L(\mathcal{A}) \subseteq \Sigma^{\omega}$ be a non self dual Borel set accepted by a (real time) Büchi 1-counter automaton $\mathcal{A}$ such that $d_{w}(L) \geq \omega$.

Let $\Gamma=\Sigma \cup\{A\}$ and $R_{1} \subseteq \Gamma^{\omega} \times \Gamma^{\omega}$ be the infinitary rational relation constructed from $L(\mathcal{A})$ as in the proof of Lemma 4.3 and let

$$
R=R_{1} \cup R_{2} \subseteq \Gamma^{\omega} \times \Gamma^{\omega}
$$


The class $\mathbf{R A T} \mathbf{T}_{\omega}$ is closed under finite union therefore $R$ is an infinitary rational relation.

Lemma 4.3 and the definition of $R_{2}$ imply that $R_{\alpha}=\left\{\sigma \in \Gamma^{\omega} \mid(\sigma, \alpha) \in R\right\}$ is equal to the set $\mathcal{L}=h(L) \cup\left(h\left(\Sigma^{\omega}\right)\right)^{-}$.

Moreover, for all $u \in \Gamma^{\omega}-\{\alpha\}, R_{u}=\left\{\sigma \in \Gamma^{\omega} \mid(\sigma, u) \in R\right\}=\Gamma^{\omega}$ holds by definition of $R_{2}$.

Then $R$ may be written as the union:

$$
R=\mathcal{L} \times\{\alpha\} \bigcup \Gamma^{\omega} \times\left(\Gamma^{\omega}-\{\alpha\}\right)
$$

or

$$
R=h(L) \times\{\alpha\} \bigcup\left(h\left(\Sigma^{\omega}\right)\right)^{-} \times \Gamma^{\omega} \bigcup \Gamma^{\omega} \times\left(\Gamma^{\omega}-\{\alpha\}\right)
$$

It is easy to see that $\left(h\left(\Sigma^{\omega}\right)\right)^{-} \times \Gamma^{\omega}$ and $\Gamma^{\omega} \times\left(\Gamma^{\omega}-\{\alpha\}\right)$ are open subsets of $\Gamma^{\omega} \times \Gamma^{\omega}$. Thus $\left(h\left(\Sigma^{\omega}\right)\right)^{-} \times \Gamma^{\omega} \bigcup \Gamma^{\omega} \times\left(\Gamma^{\omega}-\{\alpha\}\right)$ is an open subset of $\Gamma^{\omega} \times \Gamma^{\omega}$. We denote $R^{\prime}$ this open set so we have $R=h(L) \times\{\alpha\} \cup R^{\prime}$.

In order to prove that $R \leq_{W} L$ it suffices to prove that $R \leq_{W} \emptyset+L$ because Lemma 4.8 states that $\emptyset+L \equiv_{W} L$. We consider the Wadge game $W(R, \emptyset+L)$. Player 1 is in charge of the set $R \subseteq \Gamma^{\omega} \times \Gamma^{\omega}$ and Player 2 is in charge of the set $\emptyset+L$.

Player 2 has a winning strategy in this game which we now describe. Whenever Player 1 "remains" in the closed set $h\left(\Sigma^{\omega}\right) \times\{\alpha\}$, i.e. whenever the word written by Player 1 is a prefix of some $\omega$-word in $h\left(\Sigma^{\omega}\right) \times\{\alpha\}$, then Player 2 follows the play of Player 1 but skipping often in such a way that he has written the word $x(1) . x(2) \cdot x(3) \ldots x(n)$ when player 1 has written the word

$$
\left(A \cdot 0 \cdot x(1) \cdot A \cdot 0^{2} \cdot x(2) \cdot A \cdot 0^{3} \cdot x(3) \cdot A \ldots A \cdot 0^{n} \cdot x(n), \alpha[3+4+5+\ldots+n+2]\right) .
$$

If Player 1 "remains" forever in the closed set $h\left(\Sigma^{\omega}\right) \times\{\alpha\}$ then after $\omega$ steps Player 1 has written an $\omega$-word $(h(x), \alpha)$ for some $x \in \Sigma^{\omega}$, and Player 2 has written $x$. So in that case $(h(x), \alpha)$ is in $R$ iff $x$ is in $L$ iff $x$ is in $\emptyset+L$.

But if at some step of the play, Player 1 "goes out of" the closed set $h\left(\Sigma^{\omega}\right) \times\{\alpha\}$, because the word he has now written is not a prefix of any $\omega$-word of $h\left(\Sigma^{\omega}\right) \times\{\alpha\}$ then its final word will be surely outside $h\left(\Sigma^{\omega}\right) \times\{\alpha\}$ hence it will be surely in $R$. Player 2 can now write a letter of $\Sigma^{\prime}-\Sigma$ in such a way that he is now like a player in charge of $\left(\Sigma^{\prime}\right)^{\omega}$ and he can now write an $\omega$-word $u \in\left(\Sigma^{\prime}\right)^{\omega}$ so that his final $\omega$-word will be in $\emptyset+L$. Thus Player 2 wins this play too.

We have then proved that $R \leq_{W} L$. 
In order to prove that $L \leq_{W} R$ we consider the function $g: \Sigma^{\omega} \rightarrow(\Sigma \cup\{A\})^{\omega} \times$ $(\Sigma \cup\{A\})^{\omega}$ defined by: $\forall x \in \Sigma^{\omega} g(x)=(h(x), \alpha)$.

It is easy to see that $g$ is continuous because $h$ is continuous. By construction it turns out that for all $\omega$-words $x \in \Sigma^{\omega},(x \in L)$ iff $((h(x), \alpha) \in R)$ iff $(g(x) \in R)$. This means that $g^{-1}(R)=L$. This implies that $L \leq_{W} R$.

Finally we have proved that $R \leq_{W} L \leq_{W} R$, so the infinitary rational relation $R$ is Wadge equivalent to the $\omega$-language $L$ and this ends the proof.

End of Proof of Theorem 4.2. Let $L \subseteq \Sigma^{\omega}$ be a Borel set accepted by a real time Büchi 1-counter automaton $\mathcal{A}$. If $L$ is non self dual, then by Lemma 4.9 there is an infinitary rational relation $R \subseteq(\Sigma \cup\{A\})^{\omega} \times(\Sigma \cup\{A\})^{\omega}$ such that $L \equiv_{W} R$. It remains to consider the case of self dual Borel sets. The alphabet $\Sigma$ being finite, a self dual Borel set $L$ is always Wadge equivalent to a Borel set in the form $\Sigma_{1} . L_{1} \cup \Sigma_{2} . L_{2}$, where $\left(\Sigma_{1}, \Sigma_{2}\right)$ form a partition of $\Sigma$, and $L_{1}, L_{2} \subseteq \Sigma^{\omega}$ are non self dual Borel sets such that $L_{1} \equiv_{W} L_{2}^{-}$. Moreover $L_{1}$ and $L_{2}$ can be taken in the form $L_{\left(u_{1}\right)}=u_{1} . \Sigma^{\omega} \cap L$ and $L_{\left(u_{2}\right)}=u_{2} . \Sigma^{\omega} \cap L$ for some $u_{1}, u_{2} \in \Sigma^{\star}$, see Dup03]. So if $L \subseteq \Sigma^{\omega}$ is a self dual Borel set accepted by a real time Büchi 1-counter automaton then $L \equiv_{W} \Sigma_{1} \cdot L_{1} \cup \Sigma_{2} . L_{2}$, where $\left(\Sigma_{1}, \Sigma_{2}\right)$ form a partition of $\Sigma$, and $L_{1}, L_{2} \subseteq \Sigma^{\omega}$ are non self dual Borel sets accepted by real time Büchi 1-counter automata. We have already proved that there is an infinitary rational relation $T_{1} \subseteq \Gamma^{\omega} \times \Gamma^{\omega}$ such that $T_{1} \equiv_{W} L_{1}$ and an infinitary rational relation $T_{2} \subseteq \Gamma^{\omega} \times \Gamma^{\omega}$ such that $T_{2} \equiv_{W} L_{2}$. Thus $L \equiv_{W} \Sigma_{1} \cdot L_{1} \cup \Sigma_{2} \cdot L_{2} \equiv_{W} \Gamma_{1} \cdot T_{1} \cup \Gamma_{2} \cdot T_{2}$, where $T_{1}$ and $T_{2}$ are subsets of $\Gamma^{\omega} \times \Gamma^{\omega}$ and $\left(\Gamma_{1}, \Gamma_{2}\right)$ form a partition of $\Gamma \times \Gamma$. Moreover $\Gamma_{1} \cdot T_{1} \cup \Gamma_{2} \cdot T_{2}$ is an infinitary rational relation.

The Wadge hierarchy is a (great) refinement of the Borel hierarchy and, for each countable non null ordinal $\gamma, \boldsymbol{\Sigma}_{\gamma}^{0}$-complete sets (respectively, $\boldsymbol{\Pi}_{\gamma}^{0}$-complete sets) form a single equivalence class of $\equiv_{W}$, i.e. a single Wadge degree, Wad83, Kec95]. Thus we can state the following result which is a direct consequence of above Theorem 4.2 and of [Fin06a, Theorem 5.7].

\section{Corollary 4.10.}

(a) The Borel hierarchy of the class $\mathbf{R A T}_{\omega}$ is equal to the Borel hierarchy of the class $\Sigma_{1}^{1}$.

(b) $\gamma_{2}^{1}$ is the supremum of the set of Borel ranks of infinitary rational relations.

(c) For every non null ordinal $\alpha<\omega_{1}^{\mathrm{CK}}$, there exists some $\boldsymbol{\Sigma}_{\alpha}^{0}$-complete and some $\boldsymbol{\Pi}_{\alpha}^{0}$-complete infinitary rational relations.

\section{Concluding remarks}

We have only considered above the Wadge hierarchy of Borel sets. If we assume the axiom of $\boldsymbol{\Sigma}_{1}^{1}$-determinacy, then Theorem 4.2 can be extended by considering the class of analytic sets instead of the class of Borel sets. In fact in that case any 
set which is analytic but not Borel is $\boldsymbol{\Sigma}_{1}^{1}$-complete, see Kec95. So there is only one more Wadge degree containing $\boldsymbol{\Sigma}_{1}^{1}$-complete sets. We had already proved in Fin03b that there is a $\boldsymbol{\Sigma}_{1}^{1}$-complete set accepted by a 2-tape Büchi automaton.

It is natural to ask for decidability results like : "Is there an algorithm to determine the Wadge degree of a given infinitary rational relation accepted by a given 2-tape Büchi automaton?". In the case of (1-tape) automata the existence of such an algorithm has been proved by Wagner Wag79.

Unfortunately this is not possible in the case of infinitary rational relations accepted by 2-tape Büchi automata. We proved in Fin03d the following undecidability result :

Theorem 5.1 ( Fin03d $)$. Let $\Sigma$ and $\Gamma$ be finite alphabets having at least two letters, and $\alpha$ be a countable ordinal $\geq 1$. Then for an effectively given infinitary rational relation $R \subseteq \Sigma^{\omega} \times \Gamma^{\omega}$ it is undecidable to determine whether:

(a) $R$ is in the Borel class $\boldsymbol{\Sigma}_{\alpha}^{\mathbf{0}}$.

(b) $R$ is in the Borel class $\boldsymbol{\Pi}_{\alpha}^{\mathbf{0}}$.

(c) $R$ is a Borel subset of $\Sigma^{\omega} \times \Gamma^{\omega}$.

(d) $R$ is a $\boldsymbol{\Sigma}_{1}^{\mathbf{1}}$-complete subset of $\Sigma^{\omega} \times \Gamma^{\omega}$.

This implies in particular that the Wadge hierarchy of infinitary rational relations is non effective.

\section{Acknowledgements}

Thanks to the anonymous referees for useful comments on a preliminary version of this paper.

\section{References}

[BCPS03] M.-P. Béal, O. Carton, C. Prieur, and J. Sakarovitch. Squaring transducers: an efficient procedure for deciding functionality and sequentiality. Theoretical Computer Science, 292(1):45-63, 2003.

[Ber79] J. Berstel. Transductions and context free languages. Teubner Studienbücher Informatik, 1979.

[BT70] Ya M. Barzdin and B.A. Trakhtenbrot. Finite automata, behaviour and synthesis. Nauka, Moscow, 1970. English translation, North Holland, Amsterdam, 1973.

[Büc62] J.R. Büchi. On a decision method in restricted second order arithmetic. In Stanford University Press, editor, Proceedings of the 1960 International Congress on Logic Methodology and Philosophy of Science, pages 1-11. Stanford University Press, 1962. 
[CG99] C. Choffrut and S. Grigorieff. Uniformization of rational relations. In Juhani Karhumäki, Hermann A. Maurer, Gheorghe Paun, and Grzegorz Rozenberg, editors, Jewels are Forever, Contributions on Theoretical Computer Science in Honor of Arto Salomaa, pages 59-71. Springer, 1999.

[CP97] O. Carton and D. Perrin. Chains and superchains for $\omega$-rational sets, automata and semigroups. International Journal of Algebra and Computation, 7(7):673695, 1997.

[CP99] O. Carton and D. Perrin. The Wagner hierarchy of $\omega$-rational sets. International Journal of Algebra and Computation, 9(5):597-620, 1999.

[DFR01] J. Duparc, O. Finkel, and J.-P. Ressayre. Computer science and the fine structure of Borel sets. Theoretical Computer Science, 257(1-2):85-105, 2001.

[DR06] J. Duparc and M. Riss. The missing link for $\omega$-rational sets, automata, and semigroups. International Journal of Algebra and Computation, 16(1):161-186, 2006 .

[Dup01] J. Duparc. Wadge hierarchy and Veblen hierarchy: Part 1: Borel sets of finite rank. Journal of Symbolic Logic, 66(1):56-86, 2001.

[Dup03] J. Duparc. A hierarchy of deterministic context free $\omega$-languages. Theoretical Computer Science, 290(3):1253-1300, 2003.

[EH93] J Engelfriet and H. J. Hoogeboom. X-automata on $\omega$-words. Theoretical Computer Science, 110(1):1-51, 1993.

[Fin01] O. Finkel. An effective extension of the Wagner hierarchy to blind counter automata. In Proceedings of Computer Science Logic, 15th International Workshop, CSL 2001, volume 2142 of Lecture Notes in Computer Science, pages 369-383. Springer, 2001.

[Fin03a] O. Finkel. On infinitary rational relations and Borel sets. In Discrete Mathematics and Theoretical Computer Science, 4th International Conference, DMTCS 2003, Dijon, France, July 7-12, 2003. Proceedings, volume 2731 of Lecture Notes in Computer Science, pages 155-167. Springer, 2003.

[Fin03b] O. Finkel. On the topological complexity of infinitary rational relations. RAIRO-Theoretical Informatics and Applications, 37(2):105-113, 2003.

[Fin03c] O. Finkel. Undecidability of topological and arithmetical properties of infinitary rational relations. RAIRO-Theoretical Informatics and Applications, 37(2):115-126, 2003

[Fin05] O. Finkel. Borel ranks and Wadge degrees of context free $\omega$-languages. In Proceedings of New Computational Paradigms: First Conference on Computability in Europe, CiE 2005, Amsterdam, The Netherlands, volume 3526 of Lecture Notes in Computer Science, pages 129-138. Springer, 2005.

[Fin06a] O. Finkel. Borel ranks and Wadge degrees of omega context free languages. Mathematical Structures in Computer Science, 16(5):813-840, 2006.

[Fin06b] O. Finkel. On the accepting power of two-tape Büchi automata. In Proceedings of the 23rd International Symposium on Theoretical Aspects of Computer Science, STACS 2006, volume 3884 of Lecture Notes in Computer Science, pages 301-312. Springer, 2006.

[Fin07] O. Finkel. An example of $\Pi_{3}^{0}$-complete infinitary rational relation. Computer Science Journal of Moldova, 15(1):3-21, 2007. 
[Gir81] F. Gire. Relations rationnelles infinitaires. PhD thesis, Université Paris VII, 1981.

[Gir83] F. Gire. Une extension aux mots infinis de la notion de transduction rationelle. In Theoretical Computer Science, 6th GI-Conference, Dortmund, Germany, January 5-7, 1983, Proceedings, volume 145 of Lecture Notes in Computer Science, pages 123-139. Springer, 1983.

[GN84] F. Gire and M. Nivat. Relations rationnelles infinitaires. Calcolo, pages 91-125, 1984.

[Jec02] T. Jech. Set theory, third edition. Springer, 2002.

[Kec95] A. S. Kechris. Classical descriptive set theory. Springer-Verlag, New York, 1995.

[KMS89] A. S. Kechris, D. Marker, and R. L. Sami. $\Pi_{1}^{1}$ Borel sets. Journal of Symbolic Logic, 54(3):915-920, 1989.

[Kur66] K. Kuratowski. Topology. Academic Press, New York, 1966.

[Lan69] L.H. Landweber. Decision problems for $\omega$-automata. Mathematical Systems Theory, 3(4):376-384, 1969.

[LS77] R. Lindner and L. Staiger. Algebraische codierungstheorie - Theorie der sequentiellen codierungen. Akademie-Verlag, Berlin, 1977.

[LT94] H. Lescow and W. Thomas. Logical specifications of infinite computations. In J. W. de Bakker, Willem P. de Roever, and Grzegorz Rozenberg, editors, A Decade of Concurrency, volume 803 of Lecture Notes in Computer Science, pages 583-621. Springer, 1994.

[Mos80] Y. N. Moschovakis. Descriptive set theory. North-Holland Publishing Co., Amsterdam, 1980.

[Pin96] J.-E. Pin. Logic, semigroups and automata on words. Annals of Mathematics and Artificial Intelligence, 16:343-384, 1996.

[PP04] D. Perrin and J.-E. Pin. Infinite words, automata, semigroups, logic and games, volume 141 of Pure and Applied Mathematics. Elsevier, 2004.

[Pri00] C. Prieur. Fonctions Rationnelles de Mots Infinis et Continuité. PhD thesis, Université Paris VII, 2000.

[Sel95] V.L. Selivanov. Fine hierarchy of regular $\omega$-languages. In Proceedings of the International Joint Conference on the Theory and Practice of Software Development TAPSOFT-95, in Aarhus, Denmark, volume 915 of Lecture Notes in Computer Science, pages 277-287. Springer, 1995.

[Sel98] V.L. Selivanov. Fine hierarchy of regular $\omega$-languages,. Theoretical Computer Science, 191:37-59, 1998.

[Sel03a] V.L. Selivanov. Wadge degrees of $\omega$-languages of deterministic Turing machines. In Proceedings of the International Conference STACS 2003, 20th Annual Symposium on Theoretical Aspects of Computer Science, Berlin, Germany, volume 2607 of Lecture Notes in Computer Science, pages 97-108. Springer, 2003.

[Sel03b] V.L. Selivanov. Wadge degrees of $\omega$-languages of deterministic Turing machines. RAIRO-Theoretical Informatics and Applications, 37(1):67-83, 2003. 
[Sim92] P. Simonnet. Automates et théorie descriptive. PhD thesis, Université Paris VII, 1992.

[Sta86] L. Staiger. Hierarchies of recursive $\omega$-languages. Elektronische Informationsverarbeitung und Kybernetik, 22(5-6):219-241, 1986.

[Sta97] L. Staiger. $\omega$-languages. In Handbook of formal languages, Vol. 3, pages 339387. Springer, Berlin, 1997.

[SW78] L. Staiger and K. Wagner. Rekursive folgenmengen I. Z. Math Logik Grundlag. Math., 24:523-538, 1978.

[Tho88] W. Thomas. Automata and quantifier hierarchies. In Formal Properties of Finite Automata and Applications, LITP Spring School on Theoretical Computer Science, Ramatuelle, France, May 25-29, 1987, Proceedings, volume 386 of Lecture Notes in Computer Science, pages 104-119. Springer, 1988.

[Tho90] W. Thomas. Automata on infinite objects. In J. van Leeuwen, editor, Handbook of Theoretical Computer Science, volume B, Formal models and semantics, pages 135-191. Elsevier, 1990.

[Wad83] W. Wadge. Reducibility and determinateness in the Baire space. $\mathrm{PhD}$ thesis, University of California, Berkeley, 1983.

[Wag79] K. Wagner. On $\omega$-regular sets. Information and Control, 43(2):123-177, 1979.

Olivier Finkel

Equipe Modèles de Calcul et Complexité

Laboratoire de l'Informatique du Parallélisme

UMR 5668 - CNRS - ENS Lyon - UCB Lyon - INRIA

CNRS et Ecole Normale Supérieure de Lyon

46, Allée d'Italie 69364 Lyon Cedex 07, France.

and

Institut des Systèmes complexes

5, Rue du Vercors

69007 Lyon, France.

e-mail: Olivier.Finkel@ens-lyon.fr 